\title{
FLDS: A Comprehensive dsRNA Sequencing Method for Intracellular RNA Virus Surveillance
}

\author{
SYUN-ICHI URAYAMA ${ }^{1 *}$, YOSHIHIRO TAKAKI ${ }^{1,2}$, and TAKURO NUNOURA ${ }^{1}$ \\ ${ }^{1}$ Research and Development Center for Marine Biosciences, Japan Agency for Marine-Earth Science and Technology (JAMSTEC), \\ 2-15 Natsushima-cho, Yokosuka, Kanagawa 237-0061, Japan; and ${ }^{2}$ Department of Subsurface Geobiological Analysis and \\ Research, JAMSTEC, 2-15 Natsushima-cho, Yokosuka, Kanagawa 237-0061, Japan
}

(Received October 21, 2015-Accepted December 1, 2015-Published online February 13, 2016)

Knowledge of the distribution and diversity of RNA viruses is still limited in spite of their possible environmental and epidemiological impacts because RNA virus-specific metagenomic methods have not yet been developed. We herein constructed an effective metagenomic method for RNA viruses by targeting long double-stranded (ds)RNA in cellular organisms, which is a hallmark of infection, or the replication of dsRNA and single-stranded (ss)RNA viruses, except for retroviruses. This novel dsRNA targeting metagenomic method is characterized by an extremely high recovery rate of viral RNA sequences, the retrieval of terminal sequences, and uniform read coverage, which has not previously been reported in other metagenomic methods targeting RNA viruses. This method revealed a previously unidentified viral RNA diversity of more than 20 complete RNA viral genomes including dsRNA and ssRNA viruses associated with an environmental diatom colony. Our approach will be a powerful tool for cataloging RNA viruses associated with organisms of interest.

Key words: RNA virus, viral metagenome, dsRNA

Viruses are the universal genetic elements associated with all three domains of life (22), and virus-host interactions impact on the status of life and surrounding ecosystems (41). Historically, viruses are most often recognized as pathogens (38), and, thus, have been studied in the field of medical and crop science. Recent advances in high-throughput sequencing technologies have enabled us to identify not only viruses associated with diseases, but also those present in natural environments including oceans (41) and soil (12). Although these sequencing technologies have opened a new era in virus identification (24), a limited number of methods have been established for virus enrichment and library construction. The diversity and distribution of viruses in non-viral nucleic acid-dominant environments, such as the intracellular environments in which viruses actually replicate, still remain unclear due to technical difficulties (16). The development of a new procedure for effective virus enrichment and library construction is required in order to understand the full spectrum of diverse viruses.

RNA sequencing (RNA-seq) is a popular method in RNA virus metagenomics and is widely used for RNA virus identification (35). Purification and library construction methods have been established for RNA viruses at the extracellular stage $(7,10,40)$. However, the viral read ratio of intracellular RNA viruses (RNA viruses at the intracellular stage) in the RNA-seq library is typically $<1 \%$ because mRNA and rRNA are dominant in the total RNA fraction extracted from biological samples (25). Therefore, the enrichment of viral RNA is essential for maximizing sensitivity in the identification of novel viruses. The physical enrichment of viral particles and nuclease digestion of non-viral nucleotides has been employed

\footnotetext{
* Corresponding author. E-mail: urayamas@jamstec.go.jp;
} Tel: +81-46-867-9660; Fax: +81-46-867-9715. to increase the viral read ratio; however, a relatively low abundance of viral reads is still observed in most studies (39). These techniques are only applicable to specific RNA viruses because not all RNA viruses form viral particles (21). In addition, difficulties are associated with capturing terminal RNA sequences in an efficient and effective manner (32) and obtaining uniform coverage using the RNA-seq method. Sample preparation methods for effective viral RNA-seq are still inadequate and the sequence information generated is biased and incomplete.

In an attempt to resolve these issues, an environmental viral metagenomic approach targeting intracellular long doublestranded RNA (dsRNA) has recently been examined (2, 6, 9, 37). Intracellular dsRNA consists of the genomes of dsRNA viruses and replicative intermediates of single-stranded RNA (ssRNA) viruses, and, thus, long dsRNA is known as an RNA virus-specific molecule and molecular marker for RNA virus infection and replication (28). Therefore, a metagenomic analysis targeting intracellular long dsRNA theoretically retrieves dsRNA and ssRNA viruses, except for ssRNA retroviruses, which do not form dsRNA in the replicative stage. In addition, it is possible to eliminate non-viral nucleic acids such as mRNA and rRNA, which dominate RNA-seq reads, by DNase I, S1 nuclease, RNase, or column chromatography (44). However, previous studies have reported technical issues with the purification of dsRNA and library construction. Random priming for the reverse transcription of dsRNA does not enable the terminal sequences of the dsRNA molecule to be determined or eliminate significant contamination by non-viral sequences. The heterogeneous sequencing depth in certain viral genome segments is also an issue associated with this method $(2,6,9,37)$. Although the full-length cDNAs of dsRNA viruses may be obtained using loop primers that are ligated to the dsRNA terminal ends for reverse transcription 
(8), this method is only useful for short dsRNA viruses. Therefore, to the best of our knowledge, this method has not yet been applied to a viral metagenomic analysis.

We herein established a novel strategy to obtain full-length RNA virus sequences with extremely high efficiency by applying a short dsRNA full-length cloning method (8) for physically fragmented dsRNAs. The improved method, named FLDS (fragmented and loop primer ligated dsRNA sequencing), was applied to a diatom colony in a tide pool and revealed previously unidentified RNA viruses. Our results indicate that the diversity of environmental RNA viruses has been underestimated due to the technical limitations in identifying entire RNA viromes in cellular organisms, and this technique will be a powerful tool for cataloging RNA viruses associated with organisms of interest.

\section{Materials and Methods}

\section{Model and environmental samples}

Mycelial plugs of Magnaporthe oryzae strain S-0412-II 1a, naturally infected with Magnaporthe oryzae chrysovirus 1 strain A (MoCV1-A) (45) were incubated in $0.5 \%$ yeast extract and $2 \%$ glucose liquid broth (YG broth) with reciprocal shaking (60 rpm) at $25^{\circ} \mathrm{C}$ for 2 weeks in the laboratory of Prof. Teraoka (Tokyo University of Agriculture and Technology). Colonies of a diatom on tidal rocks in Tokyo Bay $\left(35.3405^{\circ} \mathrm{N}, 139.6396^{\circ} \mathrm{E}\right)$ were sampled in April 2014. After washing with distilled water, the colonies were stored at $-80^{\circ} \mathrm{C}$.

\section{Purification and fragmentation of $d S R N A$}

DsRNA was purified as described by Okada et al. with a few modifications $(31,46)$. Briefly, the microbial sample was disrupted in liquid nitrogen in a mortar and total nucleic acids were manually extracted. DsRNA was purified twice through a micro-spin column (empty Bio-spin column; Bio-Rad Laboratories, Inc., Hercules, CA, USA) containing cellulose powder (Cellulose D; ADVANTEC, Tokyo, Japan) to obtain pure dsRNA. The dsRNA eluted from cellulose powder in MQ water was treated with DNaseI (amplification grade, Invitrogen, Carlsbad, CA, USA) and S1 nuclease (Invitrogen) in nuclease buffer $\left(57 \mathrm{mM} \mathrm{CH}{ }_{3} \mathrm{COONa}, 9.5 \mathrm{mM} \mathrm{MgCl}_{2}, 1.9 \mathrm{mM}\right.$ $\mathrm{ZnSO}_{4}$, and $189 \mathrm{mM} \mathrm{NaCl}$ ) and was then incubated at $37^{\circ} \mathrm{C}$ for $2 \mathrm{~h}$. The final concentrations of $\mathrm{CH}_{3} \mathrm{COONa}, \mathrm{MgCl}_{2}, \mathrm{ZnSO}_{4}$, and $\mathrm{NaCl}$ were adjusted to $90 \mathrm{mM}, 15 \mathrm{mM}, 3 \mathrm{mM}$, and $300 \mathrm{mM}$, respectively. DsRNA was purified using an RNeasy Mini Kit (Qiagen, Valencia, CA). A one-tenth volume of $10 \times$ ShortCut buffer and $10 \times \mathrm{MnCl}$ provided with ShortCut RNase III (NEB Japan, Tokyo, Japan) was added to the dsRNA solution and fragmented by ultrasound at $4{ }^{\circ} \mathrm{C}$ in Snap-Cap microTUBEs using a Covaris S220 (Woburn, MA, USA). The fragmentation conditions were as follows; run time $35 \mathrm{~s}$, peak power $140.0 \mathrm{~W}$, duty factor $2.0 \%$, and 200 cycles/burst. Fragmented dsRNA was divided into two equal volumes, and maintained at $37^{\circ} \mathrm{C}$ with or without ShortCut RNase III (NEB). DsRNAs were then purified using a ZymoClean Gel RNA Recovery Kit (ZymoResearch, Orange, CA). Note that dsRNA purification from M. oryzae was carried out in the laboratory of Prof. Teraoka.

\section{cDNA synthesis and amplification for dsRNA}

The PC3-T7 loop primer (5'-p-GGA TCC CGG GAA TTC GGT AAT ACG ACT CAC TAT ATT TTT ATA GTG AGT CGT ATT A-OH-3') was ligated to fragmented dsRNA as described by Potgieter et al. (34), and dsRNA was then purified using the MinElute Gel Extraction Kit (Qiagen). After the addition of DMSO at a final concentration of $15 \%(\mathrm{v} / \mathrm{v})$, dsRNA was denatured at $95^{\circ} \mathrm{C}$ for $3 \mathrm{~min}$ and snap-frozen in ice-water slurry. RNA was reverse transcribed into cDNA from the ligated loop primer region using the Superscript III First-Strand Synthesis System (Invitrogen). After excess and hybrid RNAs were removed (34), cDNA was desalted and concentrated using the MinElute PCR cleanup kit (Qiagen). Primary cDNA strands were re-annealed by lowering the temperature from 95 to $50^{\circ} \mathrm{C}$, as described previously (30). Second strand DNA polymerization was performed using KOD-plus Neo (Toyobo, Osaka, Japan) with a primer complementary to the partial sequence of the PC3-T7 loop primer, PC2 (5'-CCG AAT TCC CGG GAT CC-3') (34). After heat activation of KOD-plus Neo in the reaction mixture provided at $96^{\circ} \mathrm{C}$ for $2 \mathrm{~min}$, template cDNA was added and incubated at $68^{\circ} \mathrm{C}$ for $5 \mathrm{~min}$. After the reaction, cDNA was amplified under the following conditions: $96^{\circ} \mathrm{C}$ for $2 \mathrm{~min}, 25$ (for MoCV1-A) or 18 (for diatom colony) cycles of $98^{\circ} \mathrm{C}$ for $10 \mathrm{~s}$, and $68^{\circ} \mathrm{C}$ for $2 \mathrm{~min}$. Small cDNA and primer dimers were removed using the $1.25 \times$ SPRIselect reagent kit (Beckman Coulter, Brea, CA, USA) according to the Left Side Size Selection procedure in the manufacturer's protocol.

\section{Total RNA extraction, cDNA synthesis, and library construction from an environmental sample}

Total RNA was isolated from a diatom colony using the TRIzol Plus RNA Purification Kit (Invitrogen) according to the manufacturer's protocol. The RNA fraction was treated with DNase I (Takara, Otsu, Japan). Double-stranded cDNA was synthesized from $2 \mu \mathrm{g}$ of total RNA with random primers (9-mers) using a PrimeScript Double Strand cDNA Synthesis Kit (Takara). The resultant cDNA was quantified using a Qubit dsDNA HS Kit.

\section{Illumina sequencing}

Ultrasound was used to fragment cDNA in Snap-Cap microTUBEs at $4^{\circ} \mathrm{C}$ using a Covaris S220 (Woburn, MA, USA). The fragmentation conditions were as follows; run time $55 \mathrm{~s}$, peak power $175.0 \mathrm{~W}$, duty factor $5.0 \%$ and 200 cycles/burst. The Illumina library was constructed with KAPA Hyper Prep Kit Illumina platforms (Kapa Biosystems, Woburn, MA, USA). The quantity of the library was evaluated using the KAPA library quantification kit (Kapa Biosystems). Each $300 \mathrm{bp}$ of the paired-end sequences of each fragment were determined with the Illumina MiSeq platform (San Diego, CA, USA).

\section{Data assembly and processing}

Raw sequence reads were processed with the CLC Genomics Workbench (CLC Bio, Aarhus, Denmark). Adaptor and primer sequences were trimmed, and low quality sequence regions were removed with default parameters. PhiX sequences derived from control libraries and experimentally contaminated sequences $(<0.05 \%$ of total reads) were also removed using a mapping tool. The consensus sequences of viral contigs were obtained de novo exclusively with the CLC Genomics Workbench (CLC Bio), and assemblies were manually examined and extended using the Tablet viewer (27). Using the mapping tool, each contig was confirmed to be constructed with at least $3 \times$ sequence coverage, $10 \times$ average coverage, and 1,000 bp in length. In cases of dominant reads (more than 10 reads) that stopped in the same position around the ends of contigs, the position was recognized as a terminal end. The predicted terminal ends of the viral genome segments were also confirmed by the presence of adjacent PCR primer sequences next to the predicted terminal sequence, except for cases of contigs with a poly(A) tail. Contigs with $70-90 \%$ nucleotide identity with other contigs were classified as the genome types of the same species. Contigs with $>90 \%$ nucleotide identity were assigned as the same genome type and only major contigs were used in further analyses. Sequences were compared against the NCBI non-redundant nucleotide and amino acid (aa) databases using BLASTN-plus and BLASTX-plus, respectively (5), and then classified by MEGAN 5.7.1. (18). A sequence analysis was performed using Genetyx-MAC software version 17.0.0 (Genetyx Corp., Tokyo, Japan) and Genetyx software version 9.1.0 (Genetyx). Most full-length small subunit rRNA sequences in the diatom colony were reconstructed from RNA-seq reads with EMIRGE (26). 


\section{Phylogenetic analysis}

Multiple alignments based on the deduced aa sequences of putative RNA-dependent RNA polymerase (RdRp) genes in dsRNA contigs were obtained using ClustalX 2.0 (23) and MEGA5 software (42). Phylogenetic analyses were conducted using MrBayes 3.2.3 (36) with the model of aa substitution, RtREV $+\mathrm{I}+\mathrm{G}+\mathrm{F}$, selected by ProtTest2.4 (1), as judged by the Akaike information criterion (33). Bayesian analyses with the covarion parameter were run with one run and four chains for 1,000,000 generations.

\section{Data accession}

The data sets supporting the results of this study are available in the GenBank database repository (accession nos. DDBJ: AP014890 AP014920) and Short Read Archive database (accession no. DDBJ: DRA003723 and DRA003724).

\section{Results}

\section{Application of FLDS to a segmented dsRNA virus}

The novel dsRNA purification and library construction method, named FLDS, consists of cellulose column chromatography, the physical fragmentation of dsRNA, cDNA synthesis using a loop primer, and the PCR amplification of cDNA (Fig. 1). The purification of dsRNA was achieved by the repeated affinity purification of dsRNA using cellulose powder and the enzymatic removal of ssRNA and DNA. Purified dsRNAs were fragmented using ultrasound to retrieve all types of dsRNA viruses in order to apply the previously reported full-length dsRNA cloning method using a loop primer (8). The full-length dsRNA cloning method requires overlapped cDNAs synthesized from both terminal ends for further cDNA amplification, and was only applicable to short

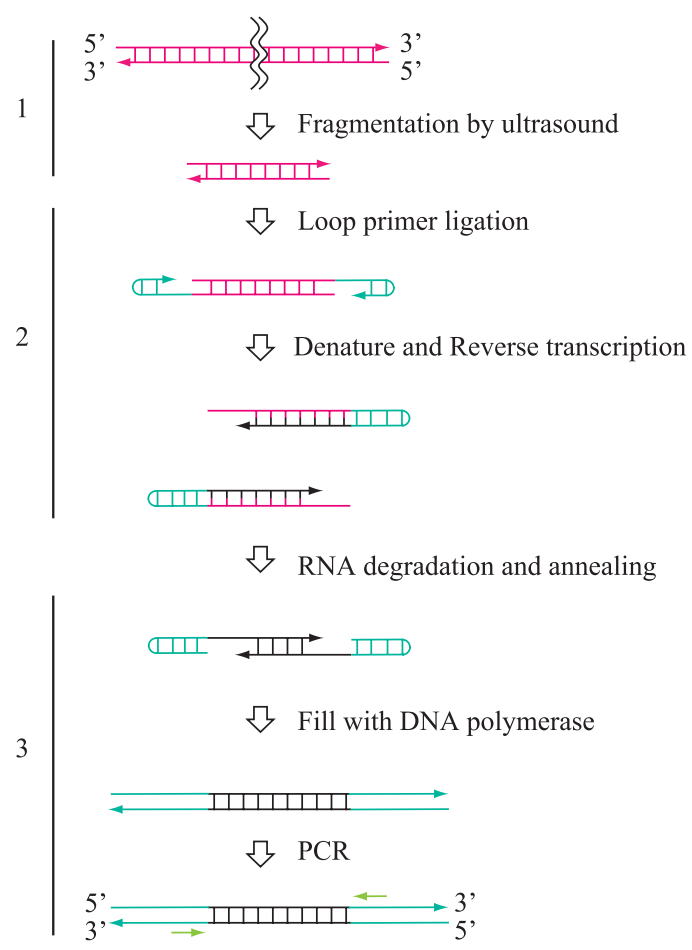

Fig. 1. Schematic work flow of FLDS. 1. Fragmentation of dsRNA by ultrasound. 2. Ligation of a loop primer on 3'-terminal ends and reverse transcription. 3. Selective duplex formation of cDNA from dsRNA, and PCR amplification. Details of the FLDS method are described in the Materials and Methods section.
dsRNA molecules. Reverse transcription was initiated from the ligated loop primer on both ends of the dsRNA fragment. cDNA was then thermally denatured to allow annealing of single-stranded cDNA with the complementary sequence in the 3 ' terminal region. The single-stranded regions of annealed cDNA were filled in with DNA polymerase. The doublestranded cDNA derived from dsRNA was amplified by PCR with a single primer (PC2) in order to obtain sufficient cDNA to construct a sequencing library.

Mycelial MoCV1-A was used to test the feasibility of this method. Since PCR amplicons were not observed in the dsRNA-specific RNaseIII-treated sample prior to reverse transcription, most of the amplicons (cDNA) were likely to have been derived from dsRNA (Fig. S1). The results of the sequencing analysis indicated that $99.1 \%$ of total reads were derived from the MoCV1-A genome (Table S1). Five contigs obtained by de novo assembly were identical to the entire region of the MoCV1-A genome segments attained using a conventional cloning and sequencing method $(44,45)$ with $>99.9 \%$ identity (Table S2). Read mapping on MoCV1-A genomes (Fig. S2) showed that the sequence coverage of terminal regions was generally higher than that of the central regions of each segment with few exceptions. No obvious relationship was observed between read coverage and GC content (Fig. S2). These results indicated that FLDS effectively enriched dsRNA reads, thereby allowing the retrieval of complete genome sequences including terminal regions without the requirement for the additional rapid amplification of cDNA ends (RACE).

\section{FLDS analysis in an environmental diatom colony}

Gel electrophoresis showed that the total long dsRNA fraction from the diatom colony contained at least ten dsRNA segments, whereas genomic DNA and rRNA were the predominant in total nucleic acids (Fig. 2). Total dsRNA extracted from $1 \mathrm{~g}$ of the diatom colony was analyzed using the FLDS method. PCR amplicons were not observed in the dsRNAspecific RNaseIII-treated sample prior to reverse transcription (Fig. S3). As a result of de novo assembly and manual extension, we obtained 42 composite viral contigs (Table 1 and Table S3). More than $98.2 \%$ of reads were mapped to these 42 contigs (Table 2) as in the case of the model experiment described above. Both terminal ends of 31 of the viral contigs were identified and recognized as full-length viral genome segments. The terminal sequences of the full-length segments were used to identify segment compositions for some of the viral species because terminal sequences are highly conserved between segments in some dsRNA viral genomes for viral RNA replication and/or encapsidation (19).

Based on aa sequence similarities (E-value $\geq 1 \times 10^{-5}$ ) in the predicted protein-encoding sequences (CDSs), the number of genome segments in related viruses, and terminal conserved sequences in each segment of a single virus, we identified 22 viral putative composite genomes out of 31 full-length viral segments. Sequence similarities between the 22 putative viral composite genomes were used to classify them into 19 putative viral species, and each of the two genome types was identified in three species (Table 1). Seventeen dsRNA and two ssRNA viral species were identified and named Diatom ColonyAssociated dsRNA virus 1-17 (DCADSRV-1-17) and Diatom 


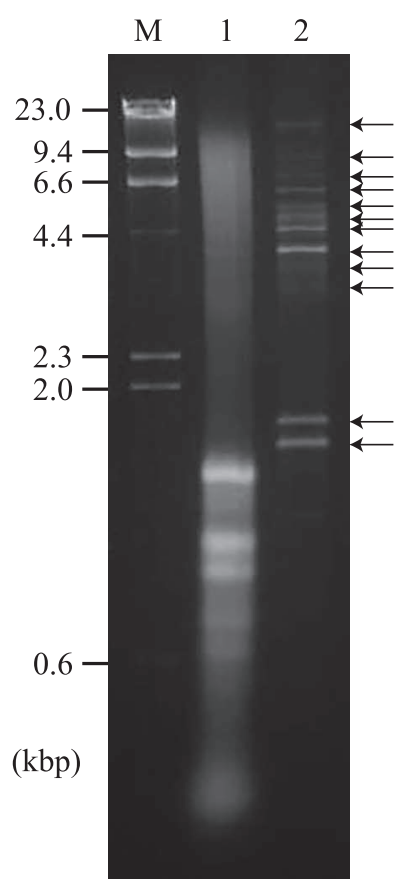

Fig. 2. Agarose gel electrophoresis of purified nucleic acids from a diatom colony. Nucleic acids were stained with ethidium bromide. Lane M, $300 \mathrm{ng}$ of HindIII-digested $\lambda$ DNA; lane 1, total nucleic acids extracted from $5 \mathrm{mg}$ (wet weight) of the diatom colony; lane 2, purified dsRNA extracted from $1 \mathrm{~g}$ (wet weight) of the diatom colony.

Colony-Associated ssRNA virus 1-2 (DCASSRV-1-2) (Table 1). Since ssRNA viruses form an RNA duplex as an intermediate in genome replication, these contigs were most likely derived from replicating ssRNA viruses (11) and not from contaminant ssRNA. An additional seven full-length viral segments with predicted CDSs were also identified; however, we were unable to determine the combination of their segments or reconstruct viral genomes based on information from previously reported viruses. Thus, these viral segments were assigned as Diatom Colony-Associated VirusLike RNA Segments (DCAVLRS-1-7).

\section{Comparison between FLDS and total RNA-seq}

Total RNA from the diatom colony was also investigated using shotgun RNA-seq in order to determine the active organisms of the colony and the abundance of viral RNA genomes in total RNA. Sequence reads derived from rRNA were identified using EMIRGE (26). The results of the analysis revealed that $56 \%$ of all trimmed reads were rRNA sequences, while $37.2 \%$ of all reads showed more than $99 \%$ identity to $18 \mathrm{~S}$ and $23 \mathrm{~S}$ rRNA from the diatom Achnanthes brevipes. In addition, 4.1 and $6.2 \%$ of reads belonged to the other diatom genus Cylindrotheca and chlorophyte genus Cladophora, respectively. The relative abundance of the rRNA reads was shown in Table S4.

Only $0.3 \%$ of reads from total RNA-seq was mapped on the major viral contigs obtained using FLDS with a read mapping algorithm in the CLC workbench (Table 2). Comparisons of the relative read frequencies of each major viral contig between total RNA-seq and FLDS revealed that FLDS achieved 0.8-4372.3-fold enrichment for each viral contig (653.2 mean) (Fig. 3). FLDS also had apparent advan- tages in uniform read coverage and efficiency for retrieving terminal sequences (Fig. 4). Sequence reads for ssRNA viruses in FLDS were also more abundant than when RNAseq was used for four out of five ssRNA contigs. In addition, by de novo assembly, only six partial viral contigs were obtained using RNA-seq, and no viral contigs specific for total RNA-seq were found. Accordingly, we concluded that FLDS is more efficient than total RNA-seq for the detection and identification of RNA viruses, with the exception of retroviruses, which theoretically cannot be identified using FLDS.

\section{Phylogenetic analysis and characterization of viral RNA genomes}

A phylogenetic analysis of viral RNA replicases (RNAdependent RNA polymerase; RdRp) presented the phylogenetic relationship between viral genomes from a diatom colony and known RNA viruses (Fig. S4). Viruses belonging to the family Totiviridae harbor non-segmented dsRNA genomes and form isometric virions that infect either fungi or protozoa (21). Thirteen composite genomes of Totiviridaerelated viruses were identified and classified into four clades distinct from the five characterized genera of Totiviridae (clades a-d in Fig. S4A). Clade c was the sister clade of the proposed genus "Trichomonasvirus" and clade d included Ustilaginoidea virens RNA virus 1 (UvRV1). In general, -1 ribosomal frameshift signals [the XXXYYYZ motif (4), in which XXX may be any three identical nucleotides, YYY may be either AAA or UUU, and Z may be A, U, or C] or +1 ribosomal frameshift signals [CCCUUUU (14) or UCCUUUCGU (47)] were located in the upstream region of the $2^{\text {nd }} \mathrm{CDS}$, and were used in the expression of overlapping viral genes such as the pol (RdRp) of Totivirus and Leishmaniavirus. These regions were examined in an attempt to better classify the identified viruses. However, as in the case of UvRV1, -1 or +1 ribosomal frameshift signals were not found in any of the Totiviridae genomes obtained in this study. CDSs in the predicted Totiviridae virus-like segments DCAVLRS-3 and DCAVLRS-4 showed significant similarities with the gag (coat protein; CP) and pol (RdRp) of known totiviruses, respectively. Totiviridae genomes consist of a single genome segment that encodes the two essential CDSs, whereas DCAVLRS-3 and -4 lacked pol and gag, respectively. These two segments harbored nine identical 5'-terminal nucleotide sequences, which were distinguishable from the other identified terminal viral sequences. Genomic features implied that DCAVLRS-3 and -4 may be parts of a bisegmented viral genome. RdRp in DCADSRV-1 segment 2 showed significant homology with that in fox Picobirnavirus, a member of the Picobirnaviridae, although DCADSRV-1 was phylogenetically distinct from the known Picobirnaviridae viruses (Fig. S4B). Picobirnaviruses are small, non-enveloped, bisegmented dsRNA viruses that infect animals and humans (21). The genome structure of DCADSRV-1 was similar to that of the known Picobirnaviridae (21). DCADSRV-14 was classified into the genus Deltapartitivirus of the family Partitiviridae based on the predicted RdRp sequence (Fig. S4C) (29). To date, all of the alphacryptoviruses have been identified from plants including the angiosperm, gymnosperm, and chlorophytes (29). rRNA sequences belonging to the Streptophyta, including land plants, have not yet been 
Table 1. List of complete composite genomes of RNA viruses and full-length virus-like RNAs obtained from a diatom colony obtained using FLDS.

\begin{tabular}{|c|c|c|c|c|c|c|c|c|}
\hline \multirow{2}{*}{$\begin{array}{l}\text { RNA virus } \\
\text { species }\end{array}$} & \multirow[b]{2}{*}{ Accession } & \multirow[b]{2}{*}{ Description } & \multirow[b]{2}{*}{ Size (nt) } & \multirow{2}{*}{$\begin{array}{l}\text { Num. of } \\
\text { mapped } \\
\text { reads }\end{array}$} & \multirow{2}{*}{$\begin{array}{l}\text { Average } \\
\text { coverage }\end{array}$} & \multicolumn{3}{|l|}{ Blast $\mathrm{X}$ analysis } \\
\hline & & & & & & Top Hit for each CDS, Virus family & E-value & Protein \\
\hline \multirow{2}{*}{ DCADSRV-1 ${ }^{\text {a) }}$} & AP014890 & segment 1 & 1,734 & $1,301,278$ & 191,942 & - & - & \\
\hline & AP014891 & & 1,562 & $1,717,396$ & 279,580 & $\begin{array}{r}\text { Fox Picobirnavirus } \\
\text { Picobirnaviridae }\end{array}$ & $1 \times 10^{-33}$ & $\mathrm{RdRp}$ \\
\hline DCADSRV-2 & AP014892 & & 4,026 & $1,337,570$ & 83,876 & $\begin{array}{r}\text { Ustilaginoidea virens nonsegmented virus } 1 \\
\text { Not assigned }\end{array}$ & $5 \times 10^{-15}$ & $\mathrm{RdRp}$ \\
\hline DCADSRV-3 & AP014893 & & 4,911 & 14,544 & 703 & $\begin{array}{r}\text { Ustilaginoidea virens } \text { RNA virus } 1 \\
\text { Totiviridae }\end{array}$ & $2 \times 10^{-63}$ & $\mathrm{RdRp}$ \\
\hline DCADSRV-4 & AP014894 & Genome type A & 4,982 & 12,325 & 591 & $\begin{array}{r}\text { Aspergillus mycovirus } 178 \\
\text { Totiviridae }\end{array}$ & $4 \times 10^{-69}$ & $\mathrm{RdRp}$ \\
\hline DCADSRV-4 & AP014895 & Genome type B & 4,979 & 1,074 & 52 & $\begin{array}{r}\text { Ustilaginoidea virens } \text { RNA virus } 1 \\
\text { Totiviridae }\end{array}$ & $5 \times 10^{-69}$ & $\operatorname{RdRp}$ \\
\hline DCADSRV-5 & AP014896 & & 5,252 & 7,863 & 359 & $\begin{array}{r}\text { Aspergillus foetidus slow virus } 1 \\
\text { Totiviridae }\end{array}$ & $3 \times 10^{-74}$ & $\mathrm{RdRp}$ \\
\hline DCADSRV-6 & AP014897 & & 4,939 & 2,720 & 131 & $\begin{array}{r}\text { Aspergillus mycovirus } 178 \\
\text { Totiviridae }\end{array}$ & $2 \times 10^{-66}$ & $\mathrm{RdRp}$ \\
\hline DCADSRV-7 & AP014898 & & 5,327 & 1,957 & 87 & $\begin{array}{r}\text { Gremmeniella abietina } \text { RNA virus L1 } \\
\text { Totiviridae } \\
\text { Ustilaginoidea virens } \text { RNA virus } 3 \\
\text { Totiviridae }\end{array}$ & $\begin{array}{l}3 \times 10^{-123} \\
2 \times 10^{-56}\end{array}$ & $\begin{array}{r}\mathrm{RdRp} \\
\mathrm{CP}\end{array}$ \\
\hline DCADSRV-8 & AP014899 & & 4,660 & 1,163 & 60 & $\begin{array}{r}\text { Aspergillus foetidus slow virus } 1 \\
\text { Totiviridae }\end{array}$ & $8 \times 10^{-57}$ & $\mathrm{RdRp}$ \\
\hline DCADSRV-9 & AP014900 & Genome type A & 4,844 & 1,198 & 60 & $\begin{array}{r}\text { Magnaporthe oryzae virus } 2 \\
\text { Totiviridae }\end{array}$ & $1 \times 10^{-65}$ & $\mathrm{RdRp}$ \\
\hline DCADSRV-9 & AP014901 & Genome type B & 4,845 & 364 & 18 & $\begin{array}{r}\text { Aspergillus foetidus slow virus } 1 \\
\text { Totiviridae }\end{array}$ & $2 \times 10^{-66}$ & $\mathrm{RdRp}$ \\
\hline DCADSRV-10 & AP014902 & & 5,082 & 1,244 & 59 & $\begin{array}{r}\text { Rosellinia necatrix victorivirus } 1 \\
\text { Totiviridae } \\
\text { Ustilaginoidea virens RNA virus } 1 \\
\text { Totiviridae }\end{array}$ & $\begin{array}{l}2 \times 10^{-108} \\
6 \times 10^{-50}\end{array}$ & $\begin{array}{r}\text { RdRp } \\
\text { CP }\end{array}$ \\
\hline DCADSRV-11 & AP014903 & & 5,160 & 1,173 & 55 & $\begin{array}{r}\text { Ustilaginoidea virens RNA virus } 1 \\
\text { Totiviridae } \\
\text { Ustilaginoidea virens } \text { RNA virus } 1 \\
\text { Totiviridae }\end{array}$ & $\begin{array}{l}4 \times 10^{-128} \\
8 \times 10^{-64}\end{array}$ & $\begin{array}{r}\mathrm{RdRp} \\
\mathrm{CP}\end{array}$ \\
\hline DCADSRV-12 & AP014904 & & 5,941 & 1,219 & 49 & $\begin{array}{r}\text { Beauveria bassiana } \text { RNA virus } 1 \\
\text { Totiviridae }\end{array}$ & $1 \times 10^{-40}$ & $\mathrm{RdRp}$ \\
\hline DCADSRV-13 & AP014905 & & 4,671 & 820 & 42 & $\begin{array}{r}\text { Aspergillus foetidus } \text { slow virus } 1 \\
\text { Totiviridae }\end{array}$ & $4 \times 10^{-58}$ & $\overline{R d R p}$ \\
\hline \multirow[t]{2}{*}{ DCADSRV-14a) } & AP014906 & segment 1 & 1,576 & 438 & 67 & $\begin{array}{r}\text { Persimmon cryptic virus } \\
\text { Partitiviridae }\end{array}$ & $3 \times 10^{-97}$ & $\mathrm{RdRp}$ \\
\hline & AP014907 & & 1,490 & 274 & 43 & - & - & - \\
\hline DCADSRV-15 & AP014908 & & 12,172 & 1,482 & 29 & $\begin{array}{r}\text { Chalara endornavirus CeEV1 } \\
\text { Endornaviridae }\end{array}$ & $1 \times 10^{-115}$ & Polyprotein \\
\hline DCASSRV-1 & AP014912 & & 11,413 & 1,011 & 21 & $\begin{array}{r}\text { Border disease virus-BD31 } \\
\text { Flaviviridae }\end{array}$ & $4 \times 10^{-15}$ & Polyprotein \\
\hline DCASSRV-2 & AP014913 & & 4,586 & 4,153 & 224 & $\begin{array}{r}\text { Tuber excavatum mitovirus } \\
\text { Narnaviridae }\end{array}$ & $5 \times 10^{-20}$ & $\mathrm{RdRp}$ \\
\hline DCADSRV-16 & AP014909 & & 6,635 & 8,735 & 310 & $\begin{array}{r}\text { Rhizoctonia fumigata mycovirus } \\
\text { Not assigned }\end{array}$ & $4 \times 10^{-10}$ & $\mathrm{RdRp}$ \\
\hline DCADSRV-17 & AP014910 & Genome type A & 5,907 & 5,325 & 218 & $\begin{array}{r}\text { dsRNA virus environmental sample } \\
\text { Not assigned }\end{array}$ & $7 \times 10^{-14}$ & $\mathrm{RdRp}$ \\
\hline DCADSRV-17 & AP014911 & Genome type B & 5,909 & 1,564 & 63 & $\begin{array}{r}\text { Botrytis porri RNA virus } 1 \\
\text { Not assigned }\end{array}$ & $1 \times 10^{-13}$ & $\mathrm{RdRp}$ \\
\hline DCAVLRS-1 & AP014914 & Interrupted RdRp & 4,567 & 57,802 & 3,039 & $\begin{array}{r}\text { Ustilaginoidea virens nonsegmented virus } 1 \\
\text { Not assigned }\end{array}$ & $3 \times 10^{-11}$ & $\mathrm{RdRp}$ \\
\hline DCAVLRS-2 & AP014915 & Interrupted RdRp & 4,786 & 41,181 & 2,100 & $\begin{array}{r}\text { Ustilaginoidea virens nonsegmented virus } 1 \\
\text { Not assigned }\end{array}$ & $2 \times 10^{-11}$ & $\mathrm{RdRp}$ \\
\hline DCAVLRS-3 & AP014916 & CP only & 3,458 & 13,140 & 876 & $\begin{array}{r}\text { Ustilaginoidea virens } \text { RNA virus } 1 \\
\text { Totiviridae }\end{array}$ & $2 \times 10^{-41}$ & $\mathrm{CP}$ \\
\hline DCAVLRS-4 & AP014917 & RdRp only & 3,190 & 3,995 & 294 & $\begin{array}{r}\text { Magnaporthe oryzae virus } 2 \\
\text { Totiviridae }\end{array}$ & $2 \times 10^{-123}$ & $\mathrm{RdRp}$ \\
\hline DCAVLRS-5 & AP014918 & CP only & 3,262 & 1,331 & 96 & $\begin{array}{r}\text { Phomopsis vexans } \text { RNA virus } \\
\text { Totiviridae }\end{array}$ & $5 \times 10^{-47}$ & $\mathrm{CP}$ \\
\hline DCAVLRS-6 & AP014919 & RdRp only & 3,325 & 891 & 65 & $\begin{array}{r}\text { Ustilaginoidea virens } \begin{array}{r}\text { RNA virus } 3 \\
\text { Totiviridae }\end{array} \\
\end{array}$ & $6 \times 10^{-102}$ & $\mathrm{RdRp}$ \\
\hline DCAVLRS-7 & AP014920 & Interrupted RdRp & 1,986 & 164 & 20 & $\begin{array}{r}\text { Flammulina velutipes browning virus } \\
\text { Partitiviridae }\end{array}$ & $4 \times 10^{-63}$ & $\mathrm{RdRp}$ \\
\hline
\end{tabular}

a) The classification was based on the shared 5' terminal sequences in paired segments, whereas CDSs in the segments that did not show significant similarities with genes in databases. 
Table 2. Classification of next-generation sequencing reads obtained by FLDS and total RNA-seq.

\begin{tabular}{lcrrr}
\hline & \multicolumn{2}{c}{ FLDS } & \multicolumn{2}{c}{ total RNA-seq } \\
\cline { 2 - 5 } & Num. of reads & rate (\%) & Num. of reads & rate (\%) \\
\hline Trimmed & $4,631,738$ & 100.0 & $6,979,561$ & 100.0 \\
Major viral reads & $4,549,629$ & 98.2 & 24,036 & 0.3 \\
Unmapped reads (include minor viral reads) & 82,109 & 1.7 & $6,955,525$ & 99.6 \\
\hline
\end{tabular}

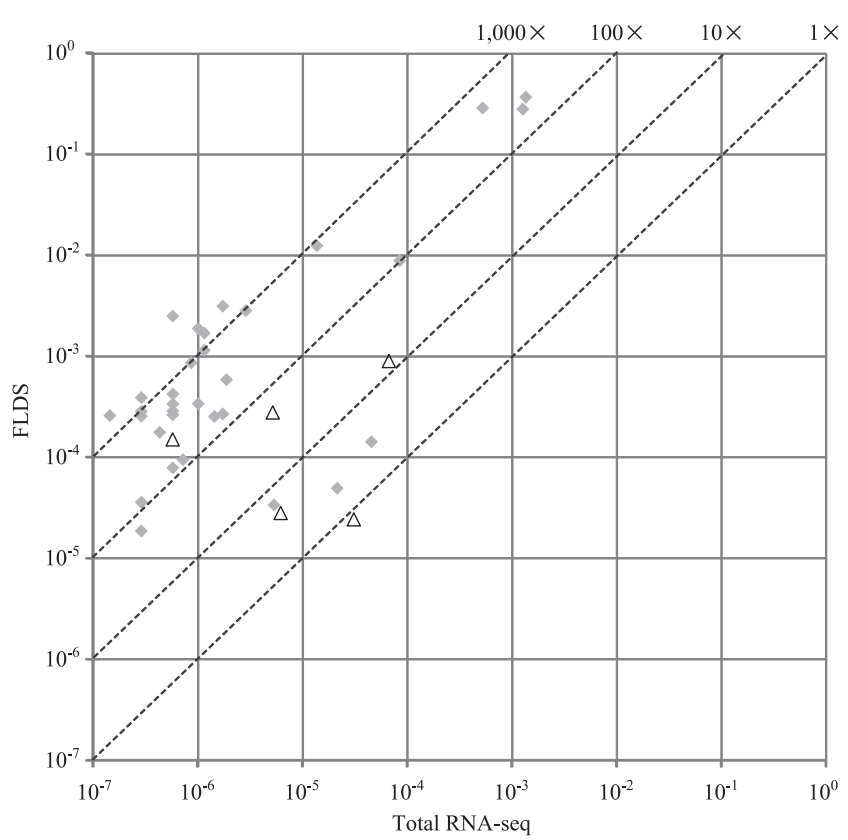

Fig. 3. Comparison of mapped read frequencies for each viral contig between FLDS and total RNA-seq. Plots indicate each viral contig. The rhombus and triangle plots show dsRNA and ssRNA viral contigs, respectively. $10^{0}-10^{-7}$ represent the frequencies of reads in each library. Dotted lines with $1 \times, 10 \times, 100 \times$, or $1000 \times$ show a higher viral read frequency than that with an RNA-seq analysis. Reads mapped with nine contigs found in FLDS were not found in total RNA-seq.

detected by an RNA-seq analysis, whereas Cladophora sp. of the Chlorophyta, a sister division of Streptophyta, were detected (Table S4). The CDSs of DCADSRV-15 and a few viral contigs showed significant homology with viruses belonging to the Endornaviridae (dsRNA), Naranviridae (ssRNA), or Hypoviridae (ssRNA), whose virion formation has not yet been observed.

In the ssRNA viral population, RdRp in DCASSRV-1 presented a close relationship with Border disease virusBD31 (E-value $\left.=4 \times 10^{-15}\right)$, a member of the genus Pestivirus of the family Flaviviridae, which consists of the arthropodborne pathogens of humans and other animals. The genome size and CDS structure of DCASSRV-1 $(11.4 \mathrm{~kb})$ were similar to those of Flaviviridae (9.6-12.3 kb) (21), and the phylogenetic tree of RdRp indicated that DCASSRV-1 was not classified into the three known Flaviviridae genera (Fig. S4D). A phylogenetic analysis of RdRp in DCASSRV-2 suggested that the RNA virus was classified into the genus Mitovirus, which has a non-segmented ssRNA genome, infects the mitochondria of fungi, and lacks viral particles (Fig. S4E). The presence of multiple UGA codons suggested that the putative coding strand of DCASSRV-2 was likely to be translated in mitochondria. The genome size of DCASSRV-2 $(4.5 \mathrm{~kb})$ was larger than those of the known mitoviruses $(2.3-3.6 \mathrm{~kb})(17)$.
(A)

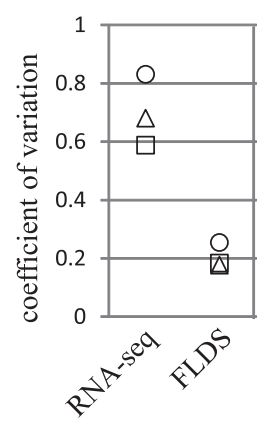

(B)

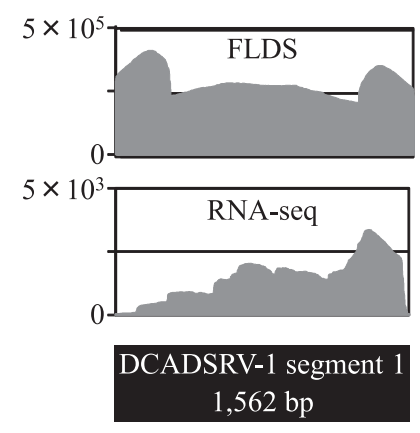

(C)

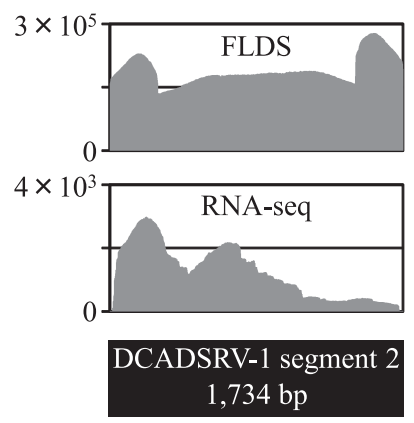

(D)

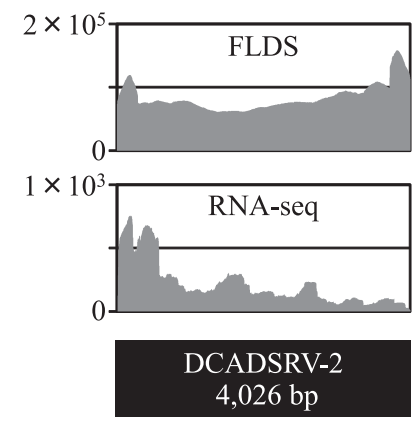

Fig. 4. Comparison of coverage uniformity between FLDS and RNAseq. DsRNA segments with an average depth of $>200$ in RNA-seq were used for the analysis. (A) Coefficient of variation (the ratio of the standard deviation to the mean coverage). Values were plotted on viral dsRNA segments of DCADSRV-1 segment 1 (square), DCADSRV-1 segment 2 (triangle), and DCADSRV-2 (circle), and were plotted on the Y axis. (B-D) Genomic coverage of each viral segment from the FLDS (upper graph) and RNA-seq (lower graph) analysis.

\section{Discussion}

This study revealed the presence of novel RNA viruses associated with a diatom colony and inferred the unexpected evolutionary relationship between environmental viruses and pathogenic animal viruses. Among the RNA viral genomes obtained in this study, some dominant populations showed greater similarities to fungal viruses than to known diatom viruses; however, several ssDNA and ssRNA viruses have already been identified from marine diatoms $(20,43)$. We cannot exclude the possibility that these viral genomes were derived from fungi associated with a diatom colony, but it is more likely that they came from the major components of a diatom colony because of their high abundance in the RNA viral metagenomic library. Since extracellular viral particles have been a major target of virus surveillance and isolation, information on intracellular viruses in microorganisms is very limited $(13,37)$. Therefore, the accumulation of knowledge on intracellular RNA viruses infecting diverse host organisms is essential for understanding the evolution and distribution of RNA viruses. 
FLDS revealed 22 full-length and some partial composite viral RNA genomes associated with a diatom colony by de novo assembly. These were classified into five dsRNA (Totiviridae, Endornaviridae, Picobirnaviridae, Cystoviridae, and Partitiviridae) and four ssRNA (Flaviviridae, Narnaviridae, Virgaviridae, and Hypoviridae) virus families. To the best of our knowledge, this is the largest number of full-length genome sequences of novel RNA viruses identified in one metagenomic library. The viral RNA community successfully detected in this study consisted of dsRNA viruses with or without virion formation and ssRNA viruses detected as replicative intermediates. Our results suggest that FLDS has the potential to detect a wide range of RNA viruses, excluding retroviruses.

Several studies have been performed using metagenomic analyses targeting dsRNA with Next-Generation Sequencing technology. In these studies, viral read abundance reached a maximum of $52.7 \%$ (2). In contrast, FLDS provided extremely high viral read abundance. The improvement in viral read rates with FLDS was likely derived from [1] a combination of repeating cellulose powder column chromatography and subsequent enzymatic treatment, [2] the fragmentation and efficient thermal denaturation of dsRNA prior to cDNA synthesis, and [3] the selective duplex formation of dsRNA-derived cDNA prior to PCR amplification. Furthermore, FLDS also presented advantages in reconstructing complete genome sequences including terminal regions, which are difficult to obtain using RNA-seq and random priming methods (3). The complete sequences of viral RNA segments are beneficial for the identification of RNA virus segments, particularly in cases in which coding CDSs did not show significant similarities with viral CDSs in databases. The application of a fulllength dsRNA cloning method using a PC3-T7 loop primer (8) to fragmented dsRNA enabled us to determine the terminal regions of long dsRNA genomes. Since T4 RNA ligase requires a $5^{\prime}$ phosphoryl-terminated nucleic acid donor (PC3T7 loop primer) and $3^{\prime}$ hydroxyl-terminated nucleic acid acceptor for ligation activity, dsRNA fragments with $3^{\prime}$ terminal phosphate were not used as substrates. The terminal structures of dsRNA fragmented by ultrasound have not been reported. However, in the case of dsDNA fragmented by ultrasound, double-strand breaks occur preferentially in $5^{\prime}-\mathrm{CpG}-3^{\prime}$ dinucleotides, and the phosphate group is at the $5^{\prime}$ side of $\mathrm{G}$ in the products (15). In this study, fragmented dsRNAs were successfully converted into cDNA and amplified. Taking this into consideration, dsRNA fragmentation using ultrasound with Covaris S220 also produced 3' hydroxylterminated fragments. Furthermore, the lack of any modifications to the 3' hydroxyl-terminal of viral RNA genomes (21) also allowed us to retrieve the terminal regions of the RNA viral genome.

Total RNA-seq is considered to be a less-biased method for identifying RNA viruses despite the very low abundance of viral reads in general. In the present study, FLDS enriched the viral RNA reads by $>300$-fold that with total RNA-seq (Table 2). Notably, FLDS produced significantly more ssRNA viral reads than total RNA-seq; however, FLDS only has the ability to detect ssRNA viruses at the replicative stage. Moreover, FLDS showed more uniform read coverage than RNA-seq. These results indicate that FLDS is more effective than total RNA-seq for revealing all RNA viruses in cellular organisms.

\section{Acknowledgements}

This research was supported in part by a Grant-in-Aid for Scientific Research (26892031) from the Ministry of Education, Culture, Sports, Science and Technology of Japan. We would like to thank Hiromitsu Moriyama, Shinsuke Kawagucci, Yukari YoshidaTakashima, and Mitsuhiro Yoshida for their fruitful discussions and valuable suggestions. We also thank Tohru Teraoka at Tokyo University of Agriculture and Technology allowing us the use of equipment.

\section{References}

1. Abascal, F., R. Zardoya, and D. Posada. 2005. Prottest: Selection of best-fit models of protein evolution. Bioinformatics 21:2104-2105.

2. Al Rwahnih, M., S. Daubert, D. Golino, and A. Rowhani. 2009. Deep sequencing analysis of RNAs from a grapevine showing Syrah decline symptoms reveals a multiple virus infection that includes a novel virus. Virology 387:395-401.

3. Alfson, K.J., M.W. Beadles, and A. Griffiths. 2014. A new approach to determining whole viral genomic sequences including termini using a single deep sequencing run. J. Virol. Methods 208:1-5.

4. Brierley, I., A.J. Jenner, and S.C. Inglis. 1992. Mutational analysis of the "slippery-sequence" component of a coronavirus ribosomal frameshifting signal. J. Mol. Biol. 227:463-479.

5. Camacho, C., G. Coulouris, V. Avagyan, N. Ma, J. Papadopoulos, K. Bealer, and T.L. Madden. 2009. Blast+: Architecture and applications. BMC Bioinformatics 10:421.

6. Coetzee, B., M.J. Freeborough, H.J. Maree, J.M. Celton, D.J. Rees, and J.T. Burger. 2010. Deep sequencing analysis of viruses infecting grapevines: Virome of a vineyard. Virology 400:157-163.

7. Culley, A.I., A.S. Lang, and C.A. Suttle. 2006. Metagenomic analysis of coastal RNA virus communities. Science 312:1795-1798.

8. Darissa, O., P. Willingmann, and G. Adam. 2010. Optimized approaches for the sequence determination of double-stranded RNA templates. J. Virol. Methods 169:397-403.

9. Decker, C.J., and R. Parker. 2014. Analysis of double-stranded RNA from microbial communities identifies double-stranded RNA viruslike elements. Cell Rep. 7:898-906.

10. Djikeng, A., R. Kuzmickas, N.G. Anderson, and D.J. Spiro. 2009. Metagenomic analysis of RNA viruses in a fresh water lake. PLoS One 4:e7264.

11. Dodds, J.A., T.J. Morris, and R.L. Jordan. 1984. Plant viral doublestranded RNA. Annu. Rev. Phytopathol. 22:151-168.

12. Fierer, N., M. Breitbart, J. Nulton, et al. 2007. Metagenomic and small-subunit rRNA analyses reveal the genetic diversity of bacteria, archaea, fungi, and viruses in soil. Appl. Environ. Microbiol. 73: 7059-7066.

13. Ghabrial, S.A., J.R. Castón, D. Jiang, M.L. Nibert, and N. Suzuki. 2015. 50-plus years of fungal viruses. Virology 479:356-368.

14. Goodman, R.P., T.S. Freret, T. Kula, et al. 2011. Clinical isolates of Trichomonas vaginalis concurrently infected by strains of up to four trichomonasvirus species (family Totiviridae). J. Virol. 85:4258-4270.

15. Grokhovsky, S.L. 2006. Specificity of DNA cleavage by ultrasound. Mol. Biol. 40:276-283.

16. Hall, R.J., J. Wang, A.K. Todd, et al. 2014. Evaluation of rapid and simple techniques for the enrichment of viruses prior to metagenomic virus discovery. J. Virol. Methods 195:194-204.

17. Hillman, B.I., and G. Cai. 2013. The family Narnaviridae: Simplest of RNA viruses. Adv. Virus Res. 86:149-176.

18. Huson, D.H., S. Mitra, H.J. Ruscheweyh, N. Weber, and S.C. Schuster. 2011. Integrative analysis of environmental sequences using MEGAN4. Genome Res. 21:1552-1560.

19. Hutchinson, E.C., J.C. von Kirchbach, J.R. Gog, and P. Digard. 2010. Genome packaging in influenza A virus. J. Gen. Virol. 91:313-328.

20. Kimura, K., and Y. Tomaru. 2015. Discovery of two novel viruses expands the diversity of single-stranded DNA and single-stranded RNA viruses infecting a cosmopolitan marine diatom. Appl. Environ. Microbiol. 81:1120-1131. 
21. King, A.M.Q., M.J. Adams, E.B. Carstens, and E.J. Lefkowitz. 2012. Virus taxonomy: Classification and nomenclature of viruses: Ninth report of the international committee on taxonomy of viruses. Elsevier.

22. Koonin, E.V. 2010. The two empires and three domains of life in the postgenomic age. Nat. Educ. 3:27.

23. Larkin, M.A., G. Blackshields, N.P. Brown, R. Chenna, P.A. McGettigan, H. McWilliam, F. Valentin, I.M. Wallace, A. Wilm, and R. Lopez. 2007. Clustal W and clustal X version 2.0. Bioinformatics 23:2947-2948.

24. Lipkin, W.I. 2013. The changing face of pathogen discovery and surveillance. Nat. Rev. Microbiol. 11:133-141.

25. Matranga, C.B., K.G. Andersen, S. Winnicki, et al. 2014. Enhanced methods for unbiased deep sequencing of Lassa and Ebola RNA viruses from clinical and biological samples. Genome Biol. 15:519.

26. Miller, C.S., B.J. Baker, B.C. Thomas, S.W. Singer, and J.F. Banfield. 2011. EMIRGE: Reconstruction of full-length ribosomal genes from microbial community short read sequencing data. Genome Biol 12:R44.

27. Milne, I., M. Bayer, L. Cardle, P. Shaw, G. Stephen, F. Wright, and D. Marshall. 2010. Tablet-next generation sequence assembly visualization. Bioinformatics 26:401-402.

28. Morris, T.J., and J.A. Dodds. 1979. Isolation and analysis of doublestranded RNA from virus-infected plant and fungal tissue. Phytopathology 69:854-858.

29. Nibert, M.L., S.A. Ghabrial, E. Maiss, T. Lesker, E.J. Vainio, D. Jiang, and N. Suzuki. 2014. Taxonomic reorganization of family Partitiviridae and other recent progress in partitivirus research. Virus Res. 188:128-141.

30. Nomikou, K., C.I. Dovas, S. Maan, S.J. Anthony, A.R. Samuel, M. Papanastassopoulou, N.S. Maan, O. Mangana, and P.P. Mertens. 2009. Evolution and phylogenetic analysis of full-length VP3 genes of eastern mediterranean bluetongue virus isolates. PLoS One 4:e6437.

31. Okada, R., E. Kiyota, H. Moriyama, T. Fukuhara, and T. Natsuaki. 2015. A simple and rapid method to purify viral dsRNA from plant and fungal tissue. J. Gen. Plant Pathol. 81:103-107.

32. Ozsolak, F., and P.M. Milos. 2011. RNA sequencing: Advances, challenges and opportunities. Nat. Rev. Genet. 12:87-98.

33. Posada, D., and T.R. Buckley. 2004. Model selection and model averaging in phylogenetics: Advantages of akaike information criterion and bayesian approaches over likelihood ratio tests. Syst. Biol. 53:793-808.

34. Potgieter, A.C., N.A. Page, J. Liebenberg, I.M. Wright, O. Landt, and A.A. van Dijk. 2009. Improved strategies for sequence-independent amplification and sequencing of viral double-stranded RNA genomes. J. Gen. Virol. 90:1423-1432.
35. Pybus, O.G., and A. Rambaut. 2009. Evolutionary analysis of the dynamics of viral infectious disease. Nat. Rev. Genet. 10:540-550.

36. Ronquist, F., and J.P. Huelsenbeck. 2003. MrBayes 3: Bayesian phylogenetic inference under mixed models. Bioinformatics 19:15721574.

37. Roossinck, M.J., P. Saha, G.B. Wiley, J. Quan, J.D. White, H. Lai, F. Chavarria, G. Shen, and B.A. Roe. 2010. Ecogenomics: Using massively parallel pyrosequencing to understand virus ecology. Mol. Ecol. 19 Suppl 1:81-88.

38. Roossinck, M.J. 2011. The good viruses: Viral mutualistic symbioses. Nat. Rev. Microbiol. 9:99-108.

39. Shah, J.D., J. Baller, Y. Zhang, K. Silverstein, Z. Xing, and C.J. Cardona. 2014. Comparison of tissue sample processing methods for harvesting the viral metagenome and a snapshot of the RNA viral community in a turkey gut. J. Virol. Methods 209:15-24.

40. Steward, G.F., A.I. Culley, J.A. Mueller, E.M. Wood-Charlson, M. Belcaid, and G. Poisson. 2013. Are we missing half of the viruses in the ocean? ISME J 7:672-679.

41. Suttle, C.A. 2005. Viruses in the sea. Nature 437:356-361.

42. Tamura, K., D. Peterson, N. Peterson, G. Stecher, M. Nei, and S. Kumar. 2011. MEGA5: Molecular evolutionary genetics analysis using maximum likelihood, evolutionary distance, and maximum parsimony methods. Mol. Biol. Evol. 28:2731-2739.

43. Tomaru, Y., K. Toyoda, H. Suzuki, T. Nagumo, K. Kimura, and Y. Takao. 2013. New single-stranded DNA virus with a unique genomic structure that infects marine diatom Chaetoceros setoensis. Scientific reports $3: 3337$

44. Urayama, S., S. Kato, Y. Suzuki, N. Aoki, M.T. Le, T. Arie, T. Teraoka, T. Fukuhara, and H. Moriyama. 2010. Mycoviruses related to chrysovirus affect vegetative growth in the rice blast fungus Magnaporthe oryzae. J. Gen. Virol. 91:3085-3094.

45. Urayama, S., T. Ohta, N. Onozuka, H. Sakoda, T. Fukuhara, T. Arie, T. Teraoka, and H. Moriyama. 2012. Characterization of Magnaporthe oryzae chrysovirus 1 structural proteins and their expression in Saccharomyces cerevisiae. J. Virol. 86:8287-8295.

46. Urayama, S., Y. Yoshida-Takashima, M. Yoshida, Y. Tomaru, H. Moriyama, K. Takai, and T. Nunoura. 2015. A new fractionation and recovery method of viral genomes based on nucleic acid composition and structure using tandem column chromatography. Microbes Environ. 30:199-203.

47. Yewdell, J.W., and W.L. Ince. 2012. Virology. Frameshifting to PA-X influenza. Science 337:164-165. 